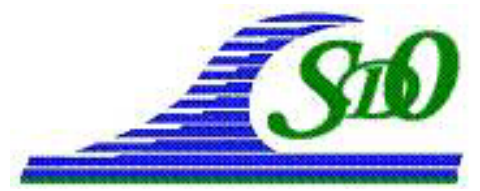

XI ìmes Journées Nationales Génie Côtier - Génie Civil

Les Sables d'Olonne, 22-25 juin 2010

DOI:10.5150/jngcgc.2010.025-D C Editions Paralia CFL

disponible en ligne - http://www.paralia.fr - available online

\title{
Réponse de la nappe d'eau sous forçage infra-gravitaire sur la plage du Truc Vert
}

\section{Olivier DAVID DE DREZIGUE ${ }^{1,2}$, Damien SOUS ${ }^{1}$, Adrien LAMBERT ${ }^{3}$, Vincent REY ${ }^{1}$}

\section{LSEET, Université de Toulon et du Var, 83957, La Garde, France.}

2. Marine nationale, Centre d'Instruction Naval de Saint-Mandrier,

Presqu'île de Saint-Mandrier, France.

Daviddedrezigue.olivier@neuf.fr

3 Géosciences Montpellier, université Montpellier 2, Montpellier, France.

\section{Résumé :}

Une série de mesures a été réalisée sur la plage du Truc Vert, Aquitaine, France, dans le cadre du programme ECORS, campagne de qualification des modèles de morphodynamique des plages sableuses. Une ligne de 18 capteurs de pression a été déployée sur un axe cross-shore de la berme au bas de la zone intertidale, combinant des capteurs immergés et enfouis dans le sable, afin de mieux caractériser la dynamique de la nappe d'eau sous différents forçages. Cette étude montre la prédominance de l'énergie dans la bande infragravitaire au niveau de la zone de surf interne et de la zone de swash, ainsi que son transfert dans l'aquifère, notamment par la propagation d'ondes de pression dans la zone saturée et la frange capillaire synchronisées avec les événements de swash.

\section{Mots-clés :}

Hydrodynamique côtière - Zone de swash - Transport sédimentaire Morphodynamique de plage

\begin{abstract}
:
As part of ECORS program, which is a qualification campaign for sandy beach morphodynamics model, an instrumental field study has been conducted on the Truc Vert beach in Aquitaine, France. A key-point of the understanding of sandy beaches dynamics is the knowledge of the interactions between surface and groundwater hydrodynamics, in particular between swash and beach watertable and their impact on accretion and erosion processes above the still water level. This study shows the infragravity energy predominance in the vicinity of the swash zone and its transfer inside the aquifer, especially by pressure waves.
\end{abstract}

Keywords:

Swash zone - Infragravity energy - Sediment process - Sandy beach morphodynamics 


\section{Introduction}

L'étude, la compréhension et la modélisation du transport sédimentaire sur les côtes à sédiments non cohésifs se révèlent être d'une importante grandissante. En effet, au-delà de l'aspect scientifique de connaissance de notre environnement, l'élévation prévisible du niveau moyen des océans dû au réchauffement climatique va, sans doute, jouer un rôle majeur dans une possible accélération de l'érosion naturelle des littoraux meubles. La nature exacte des processus contrôlant la dynamique des sédiments sur les plages sableuses n'est pas, à ce jour, complètement décrite (HORN, 2002). De nombreuses études récentes se concentrent sur l'hydrodynamique générale de la zone de swash (MASSELINK \& PUELO, 2006). La zone de swash est la partie ultime de la plage soumise au forçage de l'océan, alternativement couverte et découverte sous l'action des vagues. En particulier, les interactions entre le swash et la nappe d'eau, ou nappe phréatique, prolongement du niveau moyen de la mer dans le sable, sont supposées avoir un impact important sur les équilibres érosion/accrétion des plages (HORN, 2006). Des mesures par capteur de pression et piezomètres ont permis de mieux comprendre la dynamique de la nappe, notamment sous forçage de la marée (DAVID DE DREZIGUE et al, 2009), (RAUBENHEIMER et al, 1999) ainsi que sous forçage des vagues (TURNER \& NIELSEN, 1997).

Cette étude présente la réponse de la nappe d'eau sous un forçage infragravitaire très énergétique. L'analyse s'appuie sur des données provenant d'une campagne de mesures dans le cadre du programme PEA ECORS, sur la plage du Truc Vert (Gironde, France).

\section{Site expérimental - Instrumentation}

\subsection{Site expérimental}

La plage du Truc Vert est située à environ $10 \mathrm{~km}$ au Nord de la pointe du Cap Ferret et à $90 \mathrm{~km}$ au Sud de l'embouchure de l'estuaire de la Gironde. C'est une plage mésomacrotidale, orientée Nord-Sud, à pente douce, composée de sable moyen de diamètre environ $350 \mu \mathrm{m}$ et soumise à une houle océanique, principalement de secteur Ouest/Nord-Ouest.

La plage est généralement constituée de deux barres sableuses :

- une barre prélittorale festonnée située environ à 400-450 $\mathrm{m}$ de la laisse de basse mer. Sa longueur d'onde moyenne est de $1000 \mathrm{~m}$. La partie parallèle au rivage se situe à environ $4.5 \mathrm{~m}$ de profondeur tandis que les cornes se trouvent à $2 \mathrm{~m}$ sous l'eau.

- un système de barres/baïnes en zone intertidale dont la morphologie est très variable.

La zone intertidale (environ $200 \mathrm{~m}$ ) est suffisamment étendue pour déployer les instruments et de les récupérer à marée basse tandis qu'ils enregistrent à marée haute.

Des conditions énergétiques ont été rencontrées lors de cette campagne de mesures qui a duré 4 semaines, incluant une tempête avec des vagues de hauteurs significatives de 
$8.2 \mathrm{~m}$ et de périodes $18 \mathrm{~s}$, par une marée de $5 \mathrm{~m}$ d'amplitude. Les hauteurs significatives sont données par une bouée à houle mouillée par $20 \mathrm{~m}$ de fond, au large de la plage.

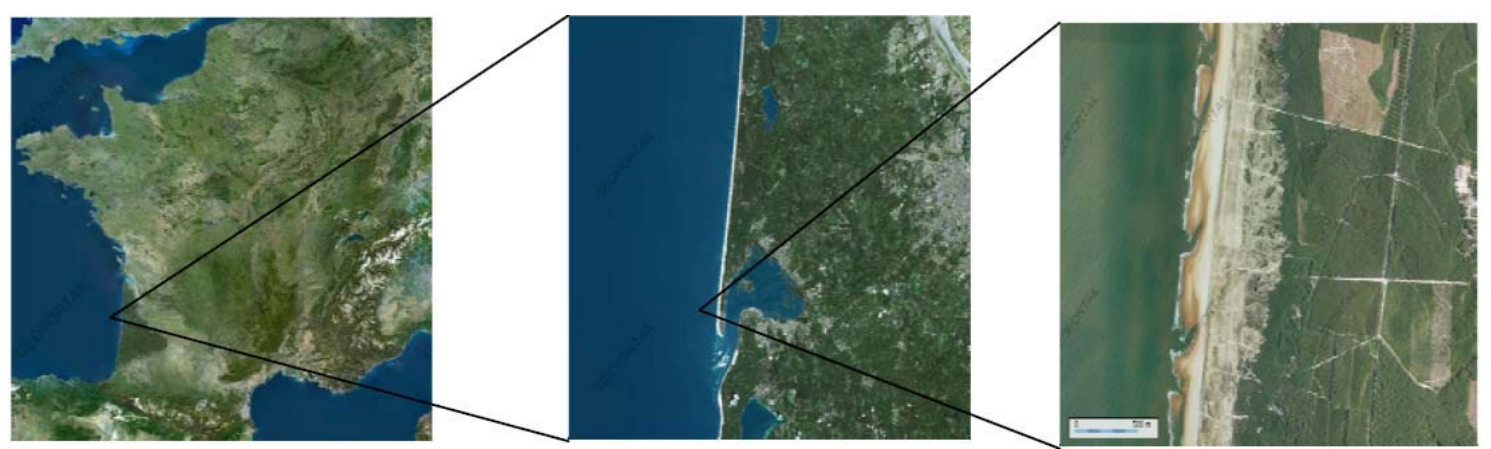

Figure 1. Emplacement géographique de la plage du Turc Vert.

\subsection{Instrumentation}

Une série de 20 capteurs a été déployée pour couvrir une section cross-shore de la plage, de la berme à la partie la plus basse de la zone intertidale. Cette ligne, alternativement immergée et émergée selon les cycles de marées, est composée de capteurs en surface et enfouis (voir figure 2) afin de permettre, simultanément, une description verticale et horizontale de la surface libre, et ce, de part et d'autre du fond sableux. Deux courantomètres acoustiques Doppler ont permis d'accéder au champ de vitesse dans le swash et la zone de surf interne, mais leurs données ne sont pas traitées dans le cadre de cette communication.

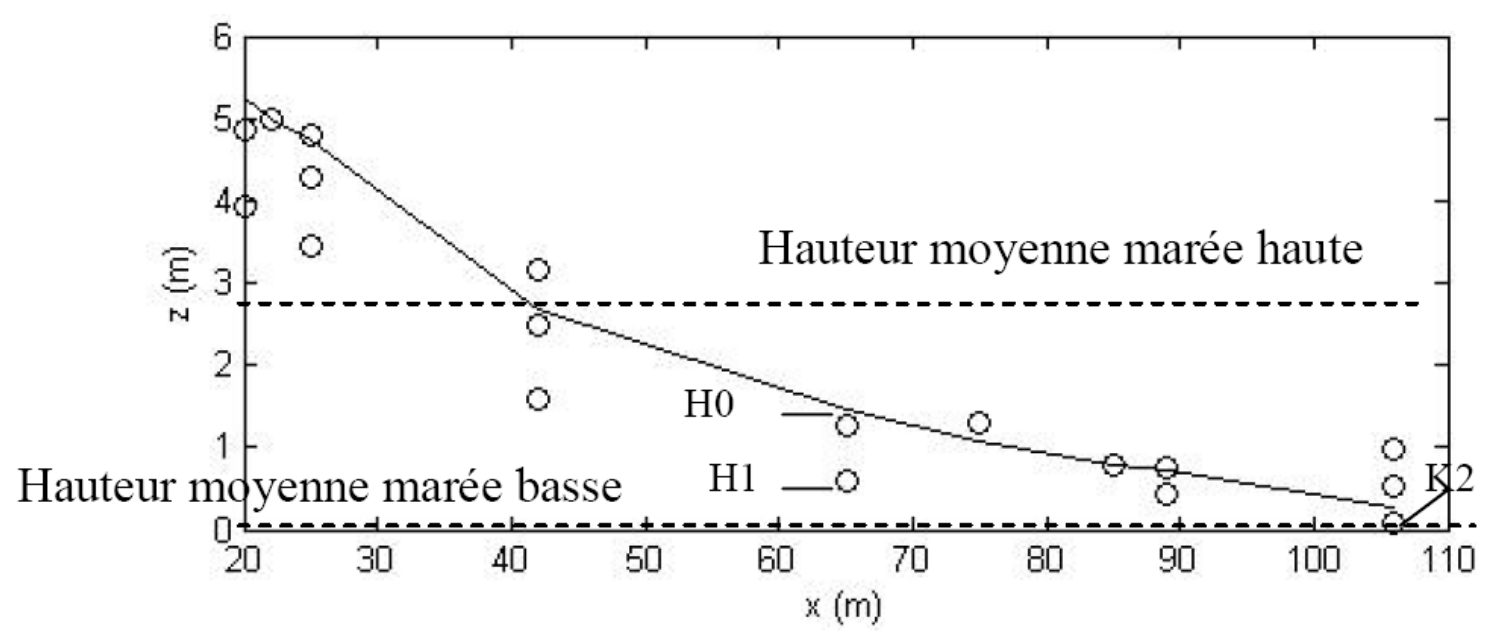

Figure 2. Profil de la plage (en trait plein) avec la disposition cross-shore de la ligne de capteurs (chaque cercle représente un capteur).

Tous les capteurs de pressions mesurent des pressions relatives, par rapport à la pression atmosphérique, et utilisent des échelles de données de 0-400 hPa ou 0-800 hPa avec une 
précision de $0.1 \%$. Ils sont protégés par un voile poreux afin d'empêcher les sédiments de s'infiltrer et de toucher les membranes. L'acquisition s'est effectuée sur la base d'un échantillonnage à $5.9 \mathrm{~Hz}$ sur toute la durée de l'étude. Les données sont enregistrées en temps réel par l'intermédiaire d'un boîtier d'acquisition, relié à un PC portable. Le positionnement des capteurs est vérifié une fois par jour, à marée basse.

Les données brutes de pression sont converties en hauteur d'eau (m) en appliquant l'équation fondamentale de l'hydrostatique :

$P=\rho g h$

où :

$P$ : pression enregistrée $(\mathrm{Pa})$

$\rho:$ masse volumique $\left(\mathrm{kg} / \mathrm{m}^{3}\right)$

$g:$ accélération gravitationnelle $\left(\mathrm{m} / \mathrm{s}^{2}\right)$

$h$ : hauteur d'eau (m)

\section{Energie infragravitaire}

Les ondes longues ou basses fréquences sont supposées jouer un rôle important dans la dynamique des plages, notamment dans le transport de particules en suspension. Les mécanismes de génération de ces ondes sont encore mal connus, cependant l'étude énergétique de la bande fréquentielle nous donne des informations intéressantes sur la présence et les caractéristiques de ces ondes. Appelées aussi ondes infragravitaires, leurs fréquences caractéristiques se situent en deçà des fréquences des ondes de gravité (houle, mer du vent), la séparation étant généralement admise à $0.05 \mathrm{~Hz}$.

Si l'énergie contenue dans la bande infragravitaire est très faible par rapport à l'énergie véhiculée par les vagues au large, cette tendance peut s'inverser à l'approche de la côte, notamment en zone de surf interne et au niveau de la zone de jet de rive ou zone de swash. La densité d'énergie de la bande infragravitaire sera ainsi prépondérante dans les mécanismes de transport de sédiments.
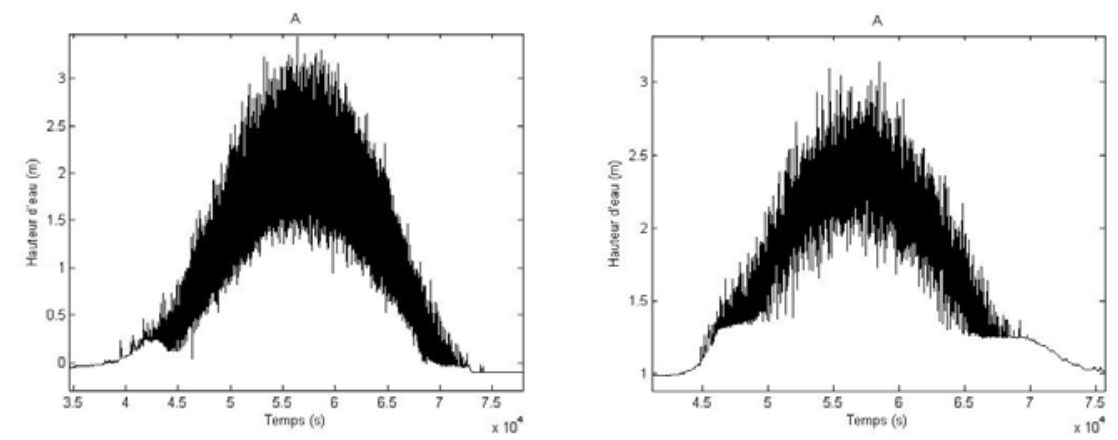

Figure 3. Signaux enregistrés par les capteurs H1 (à gauche) et K2 (à droite) sur le même cycle de marée.(Hauteur d'eau en m, en fonction du temps en s). 
La figure 3 montre les signaux convertis en hauteurs d'eau pour les capteurs $\mathrm{H} 1$ et $\mathrm{K} 2$ (voir figure 2), sur un même cycle de marée, le 22 mars 2008. Les conditions incidentes rencontrées sur ce cycle de marée sont les suivantes : hauteur significative $(\mathrm{Hs})$ de $3.2 \mathrm{~m}$, période de $10 \mathrm{~s}$. Le coefficient de marée est de 95 et l'amplitude de $4 \mathrm{~m}$. Ces deux capteurs sont distants de $41 \mathrm{~m}$. L'étude énergétique sur ces signaux permet de mieux comprendre l'évolution de la bande fréquentielle infragravitaire dans la zone de surf et de swash au cours des cycles de marée.
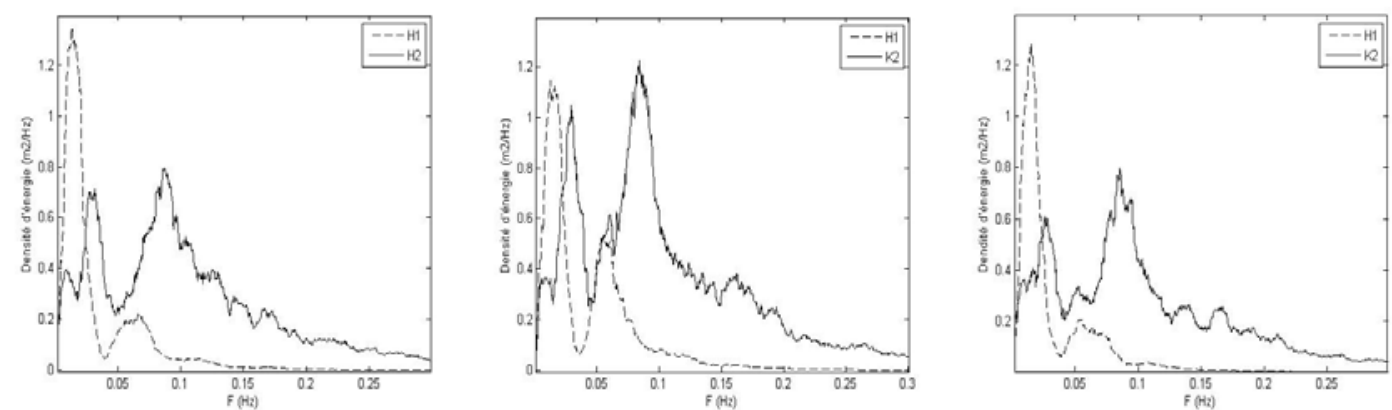

Figure 4. Densité d'énergie des signaux enregistrés sur les capteurs $H 1$ et K2, pointillés et trait plein respectivement, à trois stades distincts du cycle de marée, début de marée montante (à gauche), étale de haute mer (au milieu), fin de marée descendante (à droite). (densité spectrale d'énergie en $\mathrm{m}^{2} / \mathrm{Hz}$ en fonction de la fréquence en $\mathrm{Hz}$ ).

La figure 4 montre une étude énergétique sur les signaux enregistrés par les deux capteurs $\mathrm{H} 1$ et $\mathrm{K} 2$, à trois stades du même cycle de marée: début de marée montante, étale de marée haute et fin de marée descendante. Chaque graphe correspond à la densité spectrale $\left(\mathrm{m}^{2} / \mathrm{Hz}\right)$ du signal sur une période de calcul d'une heure.

Le creux énergétique à la fréquence de 0.05 marque bien la séparation entre le domaine des ondes longues et le domaine des vagues.

On identifie deux pics infragravitaires en $\mathrm{H} 1$ et K2, l'un autour de $0.013 \mathrm{~Hz}$ et l'autre autour de $0.027 \mathrm{~Hz}$. La composante fondamentale $(0.013 \mathrm{~Hz})$ est toujours plus énergétique que son harmonique à $0.026 \mathrm{~Hz}$. Cependant, l'énergie associée à ces deux pics varie au cours de la marée. On remarque que les situations au début de la marée montante et à la fin de la marée descendante sont similaires, avec d'avantage d'énergie associée au pic à $0.013 \mathrm{~Hz}$. A marée haute, la situation s'inverse, on observe que le pic à $0.027 \mathrm{~Hz}$ devient largement dominant. Ces observations suggèrent l'existence d'ondes pseudo-stationnaires cross-shore dont les ventres et les nœuds se déplacent avec la marée.

Si l'on considère l'énergie contenue dans la bande infragravitaire, c'est-à-dire l'intégrale de la densité d'énergie associée à la bande infragravitaire $(0.005-0.05 \mathrm{~Hz})$, et que l'on compare ces énergies entre les capteurs H1 et K2 (rapport en énergie en H1 et énergie en K2) sur des tranches horaires de différents cycles de marées, avec des conditions 
incidentes différentes, on remarque une tendance nette d'évolution de ce rapport en fonction du cycle de marée.



Figure 5. Rapport des énergies en bande infragravitaire enregistrées en H1 sur K2 sur plusieurs cycles de marée.

Sur la figure 5, chaque point représente un calcul de rapport d'énergie de bande infragravitaire en $\mathrm{H} 1$ sur K2, le trait plein la référence horaire d'un cycle de marée, et en pointillé une approximation polynomiale du nuage de points. La tendance montre, sur plusieurs cycles de marée, que ce rapport est supérieur à 1 lors du flot et du jusant, c'està-dire lorsque le capteur $\mathrm{H} 1$ est proche de la zone de swash, par contre inférieur à 1 proche de l'étale de pleine mer. Ce résultat tend à confirmer les observations réalisées précédemment sur la présence d'une onde quasi-stationnaire, probablement avec un ventre au niveau de la zone de swash, se déplaçant en suivant les variations tidales du niveau d'eau.

\section{Réponse de la nappe d'eau sous forçage infragravitaire}

On s'intéresse maintenant à la réponse de la nappe d'eau sous forçage de ces ondes longues.

La figure 6 montre les données d'enregistrement recueillies par deux capteurs de pression, $\mathrm{H} 0$ et $\mathrm{H} 1$, le capteur $\mathrm{H} 0$ se trouvant fixé sur la même barre que le capteur H1 (voire figure 2). Ces capteurs se trouvent donc à la verticale, $\mathrm{H} 0$ enfoui à $10 \mathrm{~cm}$ dans le sable et le capteur H1 à $78 \mathrm{~cm}$. Il s'agit du même cycle que précédemment, le 22 mars.

Il est intéressant d'analyser la réponse des capteurs à l'approche de l'onde de marée ainsi que de comparer les signaux entre eux à l'aide du graphe de droite de la figure 6 .

Le niveau le plus bas de la nappe est atteint autour de $\mathrm{t}=42000 \mathrm{~s}$, le capteur H1 est encore plongé dans la nappe alors que le capteur H0 est hors de la zone saturée. Si l'on regarde, en premier, le capteur $\mathrm{H} 1$, le plus profond, en trait plein : la lente et régulière montée du niveau de la nappe indique que le capteur enregistre une montée de la 
hauteur d'eau correspondant au cycle moyen de la marée, alors que les premiers swashs n'ont pas encore atteint la verticale du capteur.

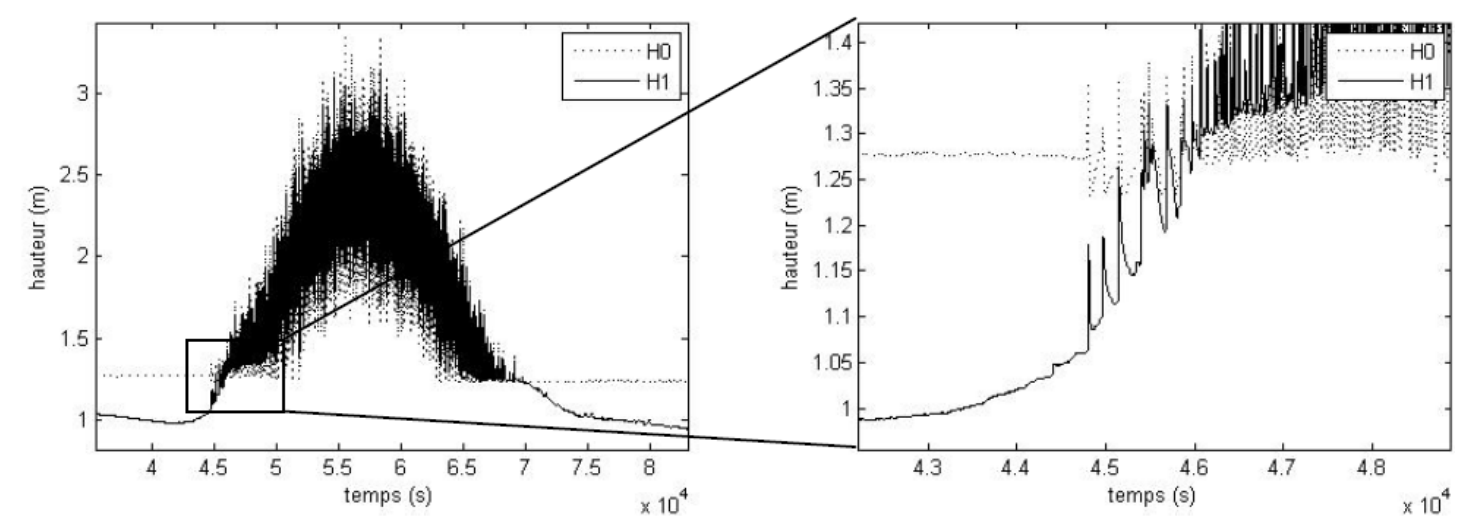

Figure 6. Enregistrement des capteurs de pression $\mathrm{HO}$ et $\mathrm{H1}$ sur un cycle de marée le 22 mars 2008 (à gauche) et zoom sur le passage du front de l'onde de marée (à droite).

Puis, en suivant la courbe, le capteur enregistre des pics de pressions nets correspondants aux premiers événements de swash, dont les périodes sont caractéristiques des ondes longues. Le sable n'est pas encore saturé sur toute la colonne, mais on observe que ces augmentations de pression sont synchronisées sur les deux capteurs superposés $\mathrm{H} 0$ et $\mathrm{H} 1$, avec des amplitudes comparables. L'origine de ces variations de pression relève probablement d'une onde de pression se propageant dans l'aquifère, plutôt que d'une élévation et une baisse rapide du toit de la nappe, a priori exclues. A l'issue de la phase de la marée montante correspondant aux premiers swashs, la colonne de sable devient saturée et le capteur quitte la zone de swash pour se retrouver en dessous de la zone de surf, et enregistre les oscillations des vagues.

Les enregistrements du capteur $\mathrm{H} 0$ sont en pointillés. La première partie de la courbe est horizontale et correspond au positionnement en " $\mathrm{z}$ " (altitude) du capteur. Le capteur enregistre bien les premiers swash des ondes longues, au même moment que le capteur H1. Cependant il est remarquable de noter qu'au retrait du swash la pression devient négative, c'est-à-dire que la pression dans le sable, au niveau du capteur, devient inférieure à la pression atmosphérique. Ce phénomène appelé "effet Wieringermeer", ou inversion de la pression est caractéristique de l'apparition d'une frange capillaire au dessus du toit de la nappe d'eau dans l'aquifère. La pression interstitielle entre les grains de sable est ainsi inférieure à la pression atmosphérique. TURNER et NIELSEN (1997) proposent une formulation pour calculer l'épaisseur de cette frange capillaire dans la zone vadose :

$H=\frac{10 \sigma}{\rho g \bar{D}}$ 
Où $\mathrm{H}$ est la hauteur de la frange capillaire, $\sigma$ la tension superficielle et $\bar{D}$ le diamètre moyen des grains de sable. L'application de la formule (2) donne une hauteur de frange de $21 \mathrm{~cm}$ (avec $\sigma=7310^{-3} \mathrm{Nm}^{-1}$ et $\bar{D}=350 \mu \mathrm{m}$ ). Si l'on calcule la distance entre le toit de la nappe (donnée par le capteur H1) et le capteur H0, sur le graphe de droite de la figure 6, au niveau des premiers swash, on trouve exactement $20 \mathrm{~cm}$, ce qui corrobore l'hypothèse de la présence d'une frange capillaire soumise à des variations de pression.

\section{Conclusion - Perspectives}

Cette communication présente les analyses d'enregistrement de pression réalisées sur la plage du Truc Vert soumise à des conditions très énergétiques. Les analyses spectrales montrent la prédominance de l'énergie dans la bande infragravitaire au niveau de la zone de surf interne et la zone de swash. Cette énergie, supposée jouer un rôle important dans les processus de transport de sédiments, est transférée dans l'aquifère, notamment par la propagation d'ondes de pression dans la zone saturée et la frange capillaire synchronisées avec les évènements de swash. Ces résultats rejoignent ceux de KIKKERT et al. (2008), et de TURNER et NIELSON (1997).

L'analyse des données en cours permettra de mieux décrire ces ondes pour permettre, à terme, d'améliorer les modèles, notamment en termes de réflexion sur des plages à pente douce et de lien avec la bathymétrie. On s'intéressera en particulier à la frange capillaire et les infiltrations/exfiltrations d'eau de mer en fonction des variations de gradients de pression.

\section{Références bibliographiques}

DAVID DE DREZIGUE O., SOUS D., LAMBERT A., GOUAUD F., REY V. (2009). Watertable response to tidal forcing in the Truc Vert sandy beach. Journal of Coastal Research. SI 56, pp 1761-1765.

HORN D.P. (2002). Beach groundwater dynamics. Geomorphology, 48, pp 121-146. doi:10.1016/S0169-555X(02)00178-2

HORN D.P. (2006). Measurements and modeling of beach groundwater flow in the swash-zone: a review. Continental Shelf Research, 26, pp 622-652. doi:10.1016/j.csr.2006.02.001

KIKKERT G., O'DONOGHUE T., PROKRAJAC D., STEENHAUER K. (2008). Effects of beach roughness on swash hydrodynamics. Coastal Engineering, pp 798-809. MASSELINK G., PUELO J.A. (2006). Swash-zone morphodynamics. Continental Shelf Research. 26, pp 661-680. doi:10.1016/j.csr.2006.01.015

RAUBENHEIMER B., GUZA R.T., ELGAR S. (1999). Tidal watertable fluctuations in a sandy ocean beach. Water Res. Res. 35, pp 2313-2320. doi:10.1029/1999WR900105

TURNER I.L., NIELSEN P. (1997). Rapid watertable fluctuations within the beach face: implications for swash zone sediment mobility? Coastal Engineering 32, pp 45-59. doi:10.1016/S0378-3839(97)00015-X 